\title{
A Proposed Experiment to Test Spin-Dependent Effects Beyond Einstein's Theory of Gravitation: The Pound-Rebka Experiment with Spin
}

\author{
J. Acosta, E. Alexeev, J. Binzer, A. Din, A. Frommelt, M. Glennon, W. Hargis, E. Langowski, H. \\ Liu, S. McKay, A. McLean, A. Mullins-Pearson, T. Payne*, M. Peters, G. Pincus, R. Reger, N. \\ Schlossberger*, L. Schnetzer, G. Schumacher, T. Scott, T. Slack, M. Starr, J. Vo, A. Walden, M. \\ Werbianskyj, M. Zhang, W.M. Snow \\ *editor
}

Faculty Mentor: Dr. W. Michael Snow, Department of Physics Indiana University

\section{ABSTRACT}

Einstein's geometric theory of gravity was constructed in part to explain why test particles in a gravitational field all follow the same trajectory independent of the mass of the particle. However, it is known that point particles in quantum mechanics must all possess at least two properties: mass and angular momentum. Many have speculated that spin-dependent effects in gravity might exist which are not contained in Einstein's theory, yet few experimental tests for such a possibility have ever been conducted. We describe an experiment which is very similar to the famous Pound-Rebka experiment, which used the Mössbauer effect to verify for the first time Einstein's prediction for the curvature of time, but which employs Mossbauer emitters and absorbers with nonzero spin. We present a specific, realistic proposal for such an experiment. We outline the theory for the "normal" effects of general relativity a la Pound-Rebka, the proposed experimental apparatus including spinpolarized emitters and absorbers, the expected sensitivity of the experiment, and potential sources of systematic error.

KEYWORDS: gravity, Mössbauer, quantum mechanics, angular momentum

\section{RATIONALE}

T The apparent conflict between our understanding of 1 gravity and quantum mechanics is one of the major unsolved problems of physics. It is perhaps not surprising that this question remains unresolved. Gravity is by far the weakest of the interactions that we presently identify as fundamental, and so far all of the available experimental data on its properties emerge from conditions in which we expect Einstein's description of gravity, in terms of a classical curved space-time with no excitation of possible quantum modes, to be valid. Our best guess for the distance scale at which one might expect effects from quantum gravity to appear, about $10^{-33} \mathrm{~m}$ as identified by Planck more than a century ago, is about 15 orders of magnitude smaller than any distance scale that has yet been directly probed in the laboratory and about eight orders of magnitude smaller than the distance scale accessible in even the highest energy cosmic rays to have been detected on Earth so far. Even the value of the gravitational constant $\mathrm{G}$ remains controversial: in a recent evaluation of $\mathrm{G}$ by the CODATA group on fundamental constants, the group assigned an uncertainty to $\mathrm{G}$ (based on the observed spread of "precision" experiments) which is only two orders of magnitude better than that measured by Cavendish two centuries ago. The irony is rich: from Newton's quantitative analysis of gravity that led to the scientific revolution and the fact that gravity is so weak, one might expect that the weakest fundamental force first isolated in physics would also be the best understood. Nothing can be further from the truth.
A natural consequence of this severe disconnect between theory and experiment in quantum gravity is the proliferation of speculative theories which give new effects that are large at the Planck scale but very small by the time the effects are extrapolated to the lower energies where we can presently do laboratory experiments. This circumstance has naturally lead theorists to analyze thought experiments on black holes in an attempt to identify, by pure thought, new fundamental principles to guide the construction of a quantum gravity theory. Much theoretical effort has also gone into the applications of quantum gravity ideas to the very early universe with the hope that some residue of these effects might survive the Big Bang and leave an imprint on observables like the cosmic microwave background. Still other approaches try to get rid of the problem by asserting that gravity is not a "real" fundamental force at all and is instead some small residual effect from one or more of the other interactions. Unfortunately, with few exceptions, these approaches have so far led to few consequences which are testable either by astronomical observation or by laboratory experiments. The recent realization that the mass energy of the universe is dominated by dark matter and dark energy comes from analysis of the dynamics of the universe at times when gravitational effects are dominant. Naturally, one might speculate that intellectual progress in our understanding of quantum gravity might have some bearing on the solution to this other major problem of physics. This revolutionary observation of dark energy has greatly expanded the number 
of scientists who really want to understand gravity: It is now not just the academic interest of a small group of theorists but also a more and more pressing issue for other researchers.

Based on our knowledge of quantum mechanics, what physics variable might be the most likely to exhibit quantum gravity effects? One popular candidate is spin. We know spin to be the most quantum mechanical of the physics properties: Turn off Planck's constant and it vanishes. We also know from Wigner's analysis of the representations of the Poincare group that an isolated particle in flat spacetime need only possess two necessary properties: mass and spin. Naturally, this idea that somehow spin should be incorporated into gravity is a very old one, dating to Cartan's ideas of spacetime torsion and continuing to the present day. Therefore, it is perhaps somewhat surprising that few gravitational experiments have been conducted which involve the measurement of spin observables.

It seems appropriate at this time to conduct more experiments involving spin and gravity for a number of reasons. First of all, the technology for producing macroscopic ensembles of polarized electrons and nuclei has greatly advanced over the last couple of decades. The number density of polarized species, the size of the polarization of the ensembles, and the delicacy with which the spins can be manipulated and measured have all greatly improved. In addition, the traditionally pessimistic theoretical estimates for the sizes of spin effects in gravity seem more and more obsolete. As an example, we consider within this context the theoretical work on the violation of CPT and Lorentz symmetry within the Standard Model Extension. This approach, which by contrast to almost all quantum gravity work takes a phenomenological approach to the problem by proposing what is basically an effective field theory for quantum gravity in the low energy limit, has identified a host of new sources for possible physical effects which have never been sought for experimentally. If there is one thing that one might guess is violated at the Planck scale, it is the CPT theorem of local quantum field theory, which is still one of the most poorly understood of the fundamental symmetries of spacetime. It is known that, under very general conditions, allowing for CPT violation forces one to also introduce the violation of Lorentz symmetry. Recently, this SME approach has been extended to gravity. While the theory is still under construction, one thing is already clear: it is quite possible that CPT/Lorentz violating gravitational effects involving the spin of particles can be quite large, contrary to previous estimates.

In this paper, we describe and analyze an experiment which could be performed to test theories which leads to spin-dependent effects in gravity. This experiment is the spin-dependent version of a famous experiment in physics: the Pound-Rebka experiment, which was used in the 1950s to measure the redshift of photons in the gravitational field of the Earth. Our proposed twist on this well-known experiment is to conduct it using polarized photons and to search for a possible photon helicity dependence to the gravitational redshift. The prediction of general relativity is that there is absolutely no such dependence. We first describe some of the key ideas behind the physics of the gravitational redshift as probed in the original experiment, which was not sensitive to the photon polarization. We then describe the modifications that would need to be made to conduct a very similar experiment with polarized photons and discuss its potential sensitivity and some of the potential sources of systematic errors that might be present. Unfortunately we are not yet able to compare this projected sensitivity with any specific theoretical predictions, which to our knowledge do not yet exist.

\section{REVIEW OF THE MÖSSBAUER EFFECT}

We first review some of the key physics ideas which one must first understand to appreciate the original Pound-Rebka experiment.

Consider an unstable nucleus with mass $m_{1}$ and energy $E_{1}$ which emits a photon. The photon is released with an energy $E_{p h}$, which is also equal to its momentum since the photon is massless. Let the energy and mass of the nucleus after the photon is emitted be $E_{2}$ and $m_{2}$ respectively. Although it is common to say that the energy of the photon is $E_{p h}=E_{1}-E_{2}$, this is not entirely accurate. When the nucleus emits a photon, it actually recoils slightly in the opposite direction that the photon was emitted. Thus we must approach the problem relativistically, such that the law of conservation of fourmomentum is not broken.

Using the known equations for relativistic energy and momentum, we can derive the correct equation for the momentum $\mathrm{p}$ due to the recoil of the nucleus and subsequently the energy $E_{p h}$ of the photon:

$$
c p=E_{p h}=\left(\frac{m_{1}^{2}-m_{2}^{2}}{2 m_{1}}\right) c^{2}
$$

Now consider briefly the implication of this result for the probability of absorption of this photon by a second nucleus. Since the energy of the emitted photon is smaller than $E_{1}-E_{2}$, this photon would not be absorbed by the second nucleus due to insufficient energy. So if the two nuclei in question are in isolated space, the second one cannot absorb the photon produced by the first one.

The Mössbauer effect refers to a modified situation in which the second nucleus can absorb the photon. This effect can occur if the nuclei in question are bound in some solid material (early work used the nucleus ${ }^{57} \mathrm{Fe}$ embedded in a solid matrix). If the atom that the nucleus is inside of is itself bound inside a solid material, then there is some nonzero probability that the recoil momentum that emits the photon is recoilless, i.e. it has no momentum in the opposite direction of the photon after emission. Technically speaking, the word "recoilless" cannot be correct as that would imply a violation of conservation of momentum. What is meant is that the 
recoil momentum is taken up collectively by all of the atoms in the material as a whole, so that the mass of the recoiling object is not the mass of the atom but rather the mass of the entire object. In the limit that the object itself has effectively infinite mass, this recoil velocity tends to zero. In practice, for a macroscopic body this recoil momentum can be so small that it is smaller than other physical effects, which leads to a slight spread in the distribution of the possible energies of the emitted photon. In this case the central value of the spread of photon energies can be centered at $E_{1}-$ $E_{2}$. Therefore the second nucleus can absorb some of these photons. This is the Mössbauer effect.

\subsection{Gravitational Redshift}

We now briefly discuss the phenomenon that the PoundRebka experiment applied the Mössbauer effect to measure, namely the gravitational redshift. The gravitational redshift consists of a shift in the energy a particle which moves between two points in a curved spacetime. The PoundRebka experiment measured for the first time the shift in photon energy between two points at different heights in the gravitational field of the Earth. The expression for the fractional shift in the photon energy between two different heights $h$ near the surface of the Earth is

$$
\frac{\delta E}{E}=\frac{g h}{c^{2}}
$$

which for a height difference of order 10 meters is of order $10^{-15}$. In the case of the $14.4 \mathrm{KeV}$ photon energy from the transition of interest in ${ }^{57} \mathrm{Fe}$, the gravitational redshift is of order $10^{-11} \mathrm{eV}$.

This shift is fantastically small. Nevertheless, it can be measured with care. We now describe some of the physical effects which must be considered to conduct such a measurement. One important effect is the spread of photon energies which comes from the finite lifetime of the excited state which produces the photon. This spread of energies possesses a width which comes from the time-energy uncertainty relation in quantum mechanics,

$$
\Delta E \Delta t \sim \hbar
$$

where $\Delta E$ is the uncertainty in energy associated with the state and $\Delta t$ is the uncertainty in time associated with the state. Using $\hbar=6.58 \times 10^{-16} \mathrm{eV} \cdot \mathrm{s}$ and the lifetime of the first excited state of $\Delta t=97.8 \mathrm{~ns}$ [10], one gets $\Delta E=7 \times 10^{-9} \mathrm{eV}$.

This is almost a factor of 1000 larger than the size of the physical effect being sought, which means that one has to be able to resolve the shift in the mean value of the photon energy to better than a part in 1000 to have a hope to see the physical effect. For practical reasons, the only way to do this in the presence of the usual sources of noise in any real experimental apparatus is to oscillate the signal of interest with a known frequency and search for the signal only at that frequency. This principle is known as lock-in detection. It greatly attenuates all sources of noise which do not possess frequency components at or near the frequency of oscillation of the effect of interest. In our case it means that we need to oscillate either the source of the absorber or the Mössbauer photons. However, the motion of the source introduces another contribution to the photon energy that must be taken into account.

This shift from the relative motion of the source and absorber can be thought of as a Doppler effect. The Doppler shift in general describes the change in frequency observed when a source is moving relative to an observer. The waves are raised in frequency if the emitter moves toward the observer, and lowered in frequency if the emitter moves away. This effect holds for light even when the emitter moves at relativistic speeds.

Consider an observer stationary in its own Frame. An emitter moves past it at a speed $\beta$ and emits a pulse of light at the start of every interval $\Delta \tau$, as measured at the emitter (staying stationary in the other Frame moving at speed $\beta$ ). For these light flashes, proper time $(\Delta \tau)$ is measured at the emitter; therefore, the time between emissions of light as seen by the observer $\left(\Delta t_{E}\right)$ is affected by relativistic time dilation and thus is given by:

$$
\Delta t_{E}=\frac{\Delta \tau}{\sqrt{1-\beta^{2}}}
$$

This is the time between emissions of the light flashes; however, this is not the time between receptions of consecutive light flashes. The emitter moves a distance of $\beta \Delta t_{E}$ between flashes in the home Frame, and so the light from the second flash will have to travel an altered distance (shorter for approaching velocities and longer for receding velocities) to reach the observer. Therefore, the time between receptions of flashes $\Delta t_{R}$ is given by:

$$
\Delta t_{R}=\Delta t_{E}+\beta \Delta t_{E}=\Delta t_{E}(1+\beta)
$$

After substitution of Eqn.(4) into Eqn.(5), this becomes:

$$
\Delta t_{R}=\frac{\Delta \tau(1+\beta)}{\sqrt{1-\beta^{2}}}=\Delta \tau \sqrt{\frac{1+\beta}{1-\beta}}
$$

This phenomenon means that the fractional shift in the photon energy becomes

$$
\frac{\delta E}{E}=\sqrt{\frac{1-\frac{v}{c}}{1+\frac{v}{c}} \cdot \frac{1-\frac{2 G M}{(R+h) c^{2}}}{1-\frac{2 G M}{R c^{2}}}}
$$

where $M$ is the mass of the Earth, $R$ is the radius of the Earth, $\mathrm{h}$ is the height above the Earth's surface, $G$ is Newton's gravitational constant, $c$ is the speed of light, and $v$ is the relative velocity between the source and the absorber. For the case of the $14.4 \mathrm{KeV}$ transition in ${ }^{57} \mathrm{Fe}$ we are using as an example, the speed needed so that the Doppler shift equals the size of the gravitational redshift is of order 


$$
v \approx \frac{g h}{c}=10^{-3} \mathrm{~cm} \cdot \mathrm{s}^{-1}
$$

which is a slow enough velocity to be varied with high precision in a practical apparatus. The idea for the measurement is then realized as follows. The source and absorber are placed at different heights in the gravitational field of the Earth. The emitter is oscillated at a constant frequency $\omega$ and with an amplitude which ensures that the Doppler effect and the gravitational redshift cancel for some point in the phase of the oscillation. At that point, the photons have the correct energy to be resonant with the absorber, and one detects the subsequent fluorescence signal from the de-excitation of the absorbed Mössbauer photons. Then the roles of the emitter and absorber are switched to isolate the gravitational redshift effect from the Doppler effect.

\section{PHYSICS ISSUES RELEVANT TO THE EXPERIMENT}

We first review some of the key physics ideas which one must first understand to appreciate the original Pound-Rebka experiment.

\subsection{Efficiency for the Mössbauer effect}

\subsubsection{Probability of recoilless emission}

The relative odds that recoilless emission of the photon occurs depend on certain properties of the nucleus and the material. The Mössbauer effect is most likely to occur if:

1. The $\gamma$-ray energy is small, and consequently $E_{R}$ is small as well.

${ }^{57} \mathrm{Fe}$ has $E \gamma=14.41 \mathrm{keV}$, which is modest compared to the energy of most nuclear $\gamma$-decays in light nuclei.

2. The temperature of the source medium is low.

Phonons in the medium can interact with the atom and cause the gamma emission energy to change such that it is not in resonance with the absorber.

3. The Debye temperature of the crystal lattice is high.

In thermodynamics and solid state physics, 2 he Debye temperature $T_{D}$ is the equivalent temperature of the crystal's highest normal mode of vibration. A higher Debye temperature means that the density of normal modes which can perturb the atoms is lower. For the ${ }^{57} \mathrm{Fe}$ source, $T_{D} \approx 470 \mathrm{~K}$. [8]

The fraction of $\gamma$-ray emissions which will be recoilless $(f)$ can be estimated using the Debye model, which assumes a continuum of oscillator frequencies to model the solid crystal lattice up to a maximum set by $\omega_{D}=k_{B} T_{D} . f$ can be expressed as:

$$
f \approx \exp \left(-\frac{E_{R}}{k_{B} T_{D}}\left\{\frac{3}{2}+\frac{\pi^{2} T^{2}}{T_{D}^{2}}\right\}\right) \quad \text { for } \quad T \ll T_{D}
$$

or,

$$
f \approx \exp \left(-\frac{6 E_{R} T}{k_{B} T_{D}^{2}}\right) \quad \text { for } \quad T>\frac{T_{D}}{2}
$$

This fraction depends on the nucleus used for the experiment. In the case of ${ }^{57} \mathrm{Fe}$, the probability of emitting a $\gamma$-ray without nuclear recoil at $0 \mathrm{~K}, 80 \mathrm{~K}$ and $300 \mathrm{~K}$ is approximately $0.93,0.92$, and 0.83 respectively. The ${ }^{57} \mathrm{Fe}$ values for $f(T)$ up to $400 \mathrm{~K}$ are plotted in Fig.(1). It is evident that, even at room temperature, a large proportion of the $\gamma$-emissions from the ${ }^{57} \mathrm{Fe}$ source will be effectively recoilless, and that cooling the source would not generate more than a $\sim 10 \%$ improvement in efficiency.

\subsubsection{Probability distribution for recoilless absorption}

The random emission process then leaves the probability to be distributed in Gaussian form.

$$
\begin{gathered}
P_{\text {absorption }} \propto \exp \left(\frac{-\chi^{2}}{2 \sigma^{2}}\right) \\
P_{\text {absorption }}\left(E, E_{\gamma}, \delta E, v, h\right)=\frac{1}{\sqrt{2 \pi \delta E^{2}}} \cdot \exp \left(-\frac{\left(E-E_{\gamma}(v, h)\right)^{2}}{2 \delta E^{2}}\right)
\end{gathered}
$$


Here, $E$ is the energy at which the a single photon is emitted, $P_{\text {absorption }}$ is the probability it is absorbed, and $E \gamma(v, h)$ is the energy of the absorbed photon in terms of its relative velocity $v$ and height $h$ described above. The emitted photon has an uncertainty in its energy $\delta E$ from the uncertainty principle due to the finite lifetime of the excited state.

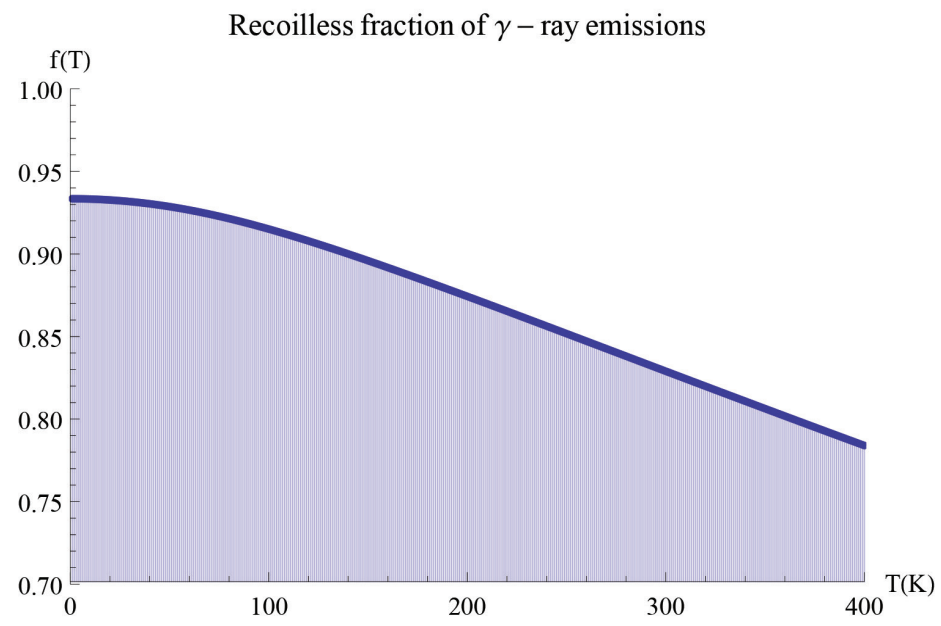

Figure 1. Temperature dependence of Mössbauer effect in ${ }^{57} \mathrm{Fe}$

\subsection{Detection of the Mössbauer absorption events}

We now consider some of the practical details relevant for the choice of the geometry of the source and absorber. Suppose we have two cylindrical samples of similar dimensions both containing excited ${ }^{57} \mathrm{Fe}$ nuclei of radius $R$ and height $h$ placed a distance $L$ apart. We will call one the "source" and the other the "absorber". The absorber is placed above the source, with both being normal to each other. Assume that the gamma rays emitted by the source through ${ }^{57} \mathrm{Fe}^{*} \rightarrow{ }^{57} \mathrm{Fe}+\mathrm{E}_{v}$ all travel a path that is normal to the surface and the absorber. The geometry is depicted in Fig.(2). The intensity at the absorber is proportional to

$$
I_{0}=\frac{\pi R^{2} A_{R}}{\pi L^{2}}
$$

where $A_{R}$ is the activity of the source.

The emitted gamma-rays will interact with ${ }^{57} \mathrm{Fe}$ nuclei in the absorber. The most prominent interactions are the Mössbauer effect (Möss), photoelectric absorption (PE), and coherent scattering (scatt); the attenuation of the photon intensity as it passes through the absorber $\delta I$ can be written as

$$
\delta I=I_{o} \exp \left(-n H \sigma_{\text {Total }}\right)
$$

where $n$ is the atomic number density, $\sigma_{\text {Total }}$ is the sum of the cross sections of the interactions mentioned above

$\left(\sigma_{\text {Total }}=\sigma_{\text {Möss }}+\sigma_{P E}+\sigma_{\text {scatt }}\right)$, and $H$ is the thickness of the absorber. Since we are interested in the gamma-rays absorbed by the absorber and then re-emitted by the Mössbauer effect, we will place our detectors adjacent to the absorber, outside of the path traveled by gamma-rays emitted by the source. We then choose $H$ to be thin enough that Mössbauer photons

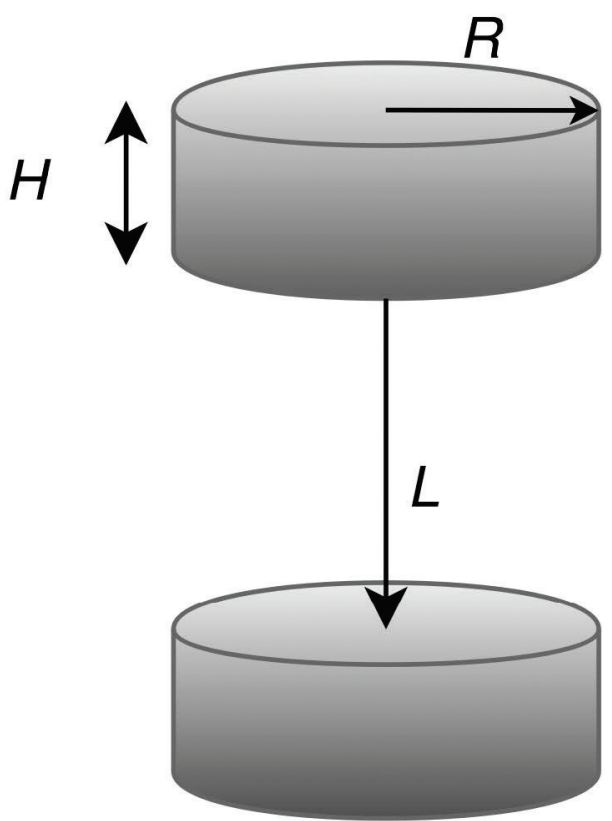

Figure 2. Geometry of the the emitter-absorber pair

will come from all nuclei in the absorber. Let us assume that our detector is some cylindrical disk flanking the absorber from all sides, as shown in Fig.(3). The size and distance of the detector is not important if we assume perfect detection and that all redirected radiation from the absorber is emitted normal to the inner surface of the detector.

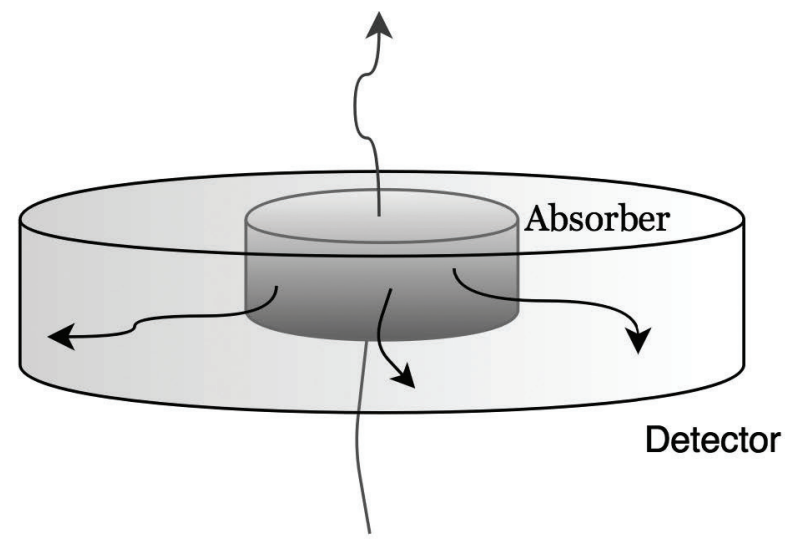

Figure 3. Depiction of the Absorber-Detector geometry

The Mössbauer intensity redirected by the absorber is proportional to

$$
I_{o}\left(1-e^{-n H \sigma_{\text {Total }}}\right) \cdot \frac{\sigma_{\text {Möss }}}{\sigma_{\text {Total }}}
$$

Assuming that on average the redirected radiation is reemitted from the center of the absorber, the intensity received by the detector is proportional to $\exp \left(-n R \sigma_{\text {Total }}\right)$.

The cross-sections will depend on the energy of the photons and the type of elemental medium in which they are traveling. 
The cross section for the photoelectric effect $\sigma_{P E}$ is given by

$$
\sigma_{\mathrm{PE}}=2 r_{o} \cdot \frac{h c}{E_{\lambda}} \cdot f_{2}
$$

where $r_{o}$ is the classical electron radius, $h$ is Planck's constant, $c$ is the speed of light, and $f_{2}$ is a complex scattering factor that can readily be found in tabulations in online databases or printed materials. The coherent Rayleigh scattering crosssection averaged over all possible collision angles can be written as

$$
\sigma_{\text {scatt }}=\frac{2}{3} \pi^{5} \cdot \frac{r^{6}}{\lambda^{4}} \cdot\left(\frac{n_{r}{ }^{2}-1}{n_{r}{ }^{2}+2}\right)^{2}
$$

$r<\lambda$, where $n_{r}$ is the index of refraction of the material, $\lambda$ is the wavelength of the photon, and $r$ is the radius of the scattering particle. Empirical and tabulated measurements of the coherent scattering cross section show it to be two orders of magnitude smaller than the photoelectric absorption cross-section in the same energy range and five orders of magnitude smaller than the Mössbauer effect cross-section for ${ }^{57} \mathrm{Fe}$; the coherent scattering cross-section could be considered negligible. We used the Lawrence Berkeley Lab's (LBL) Center for X-ray Optics' atomic scattering files [9], the National Institute of Standards and Technology's (NIST) XCOM: Photon Cross Sections Database [12] or X-Ray Form Factor, Attenuation, and Scattering Tables [14] to directly obtain the cross-sections for the photoelectric effect and for coherent scattering. The Mössbauer effect cross-section is taken from [3].

\subsection{Loss of gamma ray photon intensity between the emitter and absorber}

In this experiment, we have a few options for what the space between the emitter and receiver will contain. These options include a vacuum, hydrogen gas, and helium gas. A vacuum would allow for the maximum intensity to reach the receiver. However, a vacuum requires the presence of two material windows, so we also looked into how much gamma ray photon intensity would be lost if we were to use a gas instead. We were also interested in how much loss would occur with the rays passing through a thin, strong material which can withstand a 1 bar pressure difference. As it turns out, the aluminum used in soda cans is known to be quite strong and highly optimized for a high strength-to-thickness ratio. The essential equation to evaluate the tradeoffs for different options is the Beer-Lambert Law

$$
I=I_{o} e^{-\left(\frac{\mu}{\rho}\right) \rho l}
$$

where $(\mu / \rho)$ is the mass attenuation coefficient. Using the NIST XCOM database [13], it is easy to find this number for all three elements. The density of aluminum is well known. We used NIST's Standard Reference Database for the density of Hydrogen and Helium at 295 $\mathrm{K}$ and 1 bar.
To find how much aluminum the gamma rays would go through, we measured several soda cans and found their average width to be about $1.8 \times 10^{-4} \mathrm{~m}$. All of these parameters were plugged into the Beer-Lambert Law and the results are shown in Table (1). Out of $\mathrm{H}$ and $\mathrm{He}, \mathrm{He}$ allows for the most gamma rays to pass through with only a $0.4 \%$ intensity loss. In addition, helium gas is safe to use and is a practical choice.

\begin{tabular}{|l|c|c|c|c|}
$\mathrm{Z}$ & $\mu / \rho\left[\mathrm{m}^{2} / \mathrm{kg}\right]$ & $\rho\left[\mathrm{kg} / \mathrm{m}^{3}\right]$ & $l[\mathrm{~m}]$ & $I_{f}$ \\
\hline $\mathrm{H}$ & .03774 & .082 & 30 & $.985 I_{o}$ \\
$\mathrm{He}$ & .02116 & .16311 & 30 & $.996 I_{o}$ \\
$\mathrm{Al}$ & .8689 & 2700 & $1.8 \times 10^{-4}$ & $.158 I_{o}$
\end{tabular}

Table 1. This table shows the parameters used in the BeerLambert Law equation for hydrogen, helium, and aluminum. In its last column, it shows the fraction of the original intensity that is let through each material.

\subsection{Improving efficiency between emission and absorption using $x$-ray mirrors}

To maximize the signal received and thereby maximize data collection, it is desirable to encase the emitter, absorber, and intervening space with a mirror which will maximize the amount of photons that reach the absorber. Doing this will collect some photons which would otherwise escape through the non-reflective boundaries of the experiment, and redirect them to the absorber. X-rays reflect only at glancing angles measured from the inbound light to the surface of reflection. However, careful choice of reflective material can yield an increase in the amount of photons that reach the absorber. Wolter optics, a series of nested curved mirrors, would likely provide the best signal, but the cost of manufacturing such optics outweighs the benefit of their use in this application [17].

A more cost-effective solution is to encapsulate the experiment within a cylindrical mirror created from glass with the inner surface coated in a layer of a high-density material with high $\mathrm{x}$-ray reflectivity (illustrated in Fig. (4)). When choosing a material for coating the cylinder, maximizing the index of refraction of the material for the energy of the emitted photons $(14.4 \mathrm{keV})$ is the priority. Maximization of the index of refraction will yield a maximized angle from which photons striking the mirror will be reflected and continue to pass down the cylinder to be received by the absorber.

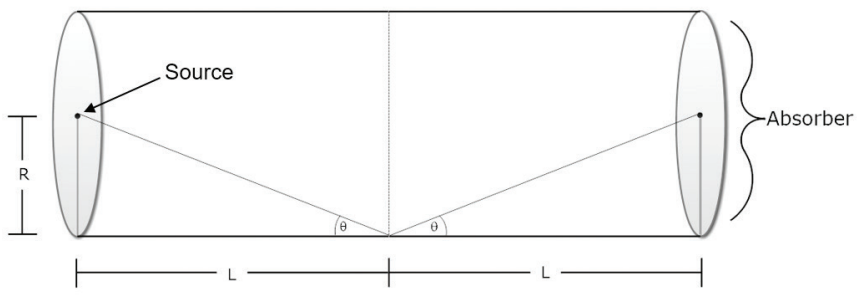

Figure 4. Geometry of mirror apparatus 
The index of refraction of a material is parametrized as:

$$
n=1-\delta-i \beta
$$

where $\delta$ is the decrement of refraction, and $\beta$ is the absorption index of the material. The index of refraction can be expressed as a function of photon energy and material by the following form:

$$
n=1-\delta-i \beta=1-\frac{r_{0} \lambda^{2} N}{2 \pi} f_{0}
$$

where $r_{0}$ is the classical electron radius, $\lambda$ is wavelength, $N$ is the atomic number density of the material, and $f_{0}$ is the atomic scattering factor, as described by Gullikson [7].

$$
f_{0}=f_{1}+i f_{2}
$$

The atomic scattering factor for a given material has both real and imaginary parts $f_{1}$ and $f_{2}$. Henke, Gullikson, and Davis have cataloged these values for all elements at photon energies ranging from $50-30,000 \mathrm{eV}$ [1].

Total external reflection occurs at a critical angle which follows from Snell's law and is given by:

$$
a_{c}=\sin ^{-1}\left(\frac{n_{2}}{n_{1}}\right)
$$

Hence, the glancing angle is:

$$
\theta=\cos ^{-1}\left(\frac{n_{2}}{n_{1}}\right)
$$

When the medium though which the approaching light is close to that of a vacuum, such as in a gas, the index of refraction of that medium is much lower than that of the mirror surface and can be treated as approximately one. In this case, the glancing angle is:

$$
\theta=\cos ^{-1}\left(n_{2}\right)
$$

The goal is to maximize the glancing angle to maximize photons received by the absorber. The chamber for the experiment should be filled with a low density uniform gas, such as the helium filled apparatus used by Pound and Rebka [15]. Assuming the index of refraction of the gas inside the chamber is approximately one, candidates for the mirror coating can be determined.

Good candidates for a reflective coating are those with a high density. Several candidates which would maximize the glancing angle are iridium and tungsten. The most effective of these choices is iridium, which would give a glancing angle of 0.0058 radians. However, tungsten gives a glancing angle of 0.0056 radians [1]. Since tungsten is significantly less expensive than iridium, it could be considered as a substitute for iridium if costs are a restraint.

The previous sections have discussed the physics of the Mössbauer effect, its use to search for the gravitational redshift from curved spacetime, the conceptual design of the experiment employing moving sources to induce a Doppler shift that cancels the gravitational effect to bring the photon energy back into resonance, and some of the technical and safety issues associated with the practical design of such an experiment. In the rest of the paper we discuss the relevant concepts needed to understand how to add photon spin to this experiment in order to test the possible spin dependence of gravity.

\subsection{Decay of ${ }^{57} \mathrm{Fe}$ source intensity}

The source intensity is proportional to the activity of the primary ${ }^{57} \mathrm{Co}$ source which feeds the excited state of interest in ${ }^{57} \mathrm{Fe}$. The equation for decay of an isotope is:

$$
N(t)=N_{o} e^{-\lambda t}
$$

where $N$ is the number of atoms of the isotope available after time, $t$, from the initial number of atoms $N_{o} . \lambda$ is the decay constant given by the half-life of the isotope, $T$, given by the equation:

$$
\lambda=\frac{\ln 2}{T}
$$

The equation for decay can also be written in terms of activity given that activity is proportional to the number of atoms $(A=N \lambda)$, so the equation for decay in activity is:

$$
A=A_{o} e^{-\lambda t}
$$

where $A_{o}$ is the initial activity of the isotope and $A$ is the activity over time, $t$. So since intensity is proportional to activity the loss of source intensity due to decay is shown by the equation:

$$
I=I_{o}\left(1-e^{\lambda t}\right)
$$

The half-life of ${ }^{57} \mathrm{Co}$ is 271.80 days [16], which is long enough to operate the experiment over a reasonable time before needing to replace the source.

\subsection{Safety precautions}

As with any experiment, it is important to understand and account for safety and health hazards associated with radioactive sources of material. Since the radiation being measured consists of X-ray photons $(14.1 \mathrm{keV})$, it is important to pick a material to safely attenuate the emissions. With its availability, density, and strong attenuation properties, lead is a good choice of material to attenuate the photons.

For 14.1 keV X-ray emissions, lead has a mass attenuation coefficient around $100 \mathrm{~cm}^{2} / \mathrm{g}$ [13]. This means for safe attenuation of the radioactive emissions (a factor of $10^{-5}$ ), the particles would need to travel through about $2 \mathrm{~mm}$ of lead. If we surround the sample and the experiment such that any X-ray emitted travels through at least this thickness of lead, the surrounding area will be effectively safe from harmful radiation. 


\section{ADDITION OF SPIN TO THE POUND-REBKA EXPERIMENT}

To explain how to generate and manipulate the photon spin angular momentum, we must first describe how one defines and manipulates the angular momentum of matter particles.

\subsection{Energy level splitting of magnetic moment in a magnetic field}

We first review the fundamental concept of quantum mechanical spin and its connection with the magnetic moments of elementary particles. A magnetic dipole moment $\mu$ is produced by current flowing in a loop, and is defined by the product of the current and the area of the loop.

Consider first a classical physics model. An object with a magnetic moment feels a torque if it is placed in an external magnetic field. The torque acting on the magnetic moment can be found by cross multiplying the magnetic moment $\mu$ and the magnetic field vector B.

$$
\vec{\tau}=\vec{\mu} \times \vec{B}
$$

The size of the magnetic moment of a charged particle is usually written as:

$$
\mu=g \frac{e \hbar}{2 m} J
$$

where $m$ is the particle's mass, $J$ is the particle's spin, $e$ is its electric charge, and $g$ is the so-called gyromagnetic ratio (which from theory is equal to 2 for a point particle with no internal structure). The ${ }^{57} \mathrm{Fe}$ nucleus has a gyromagnetic ratio of 0.1806 in its ground state [2]. Magnetic moments are often expressed in terms of the nuclear magneton $\mu_{N}$ :

$$
\mu_{N}=\frac{e \hbar}{2 m_{p}}=5.050783 \times 10^{-27} \mathrm{~J} / \mathrm{T}=3.152452 \times 10^{-8} \mathrm{eV} \cdot \mathrm{T}^{-1}
$$

For the case of ${ }^{57} \mathrm{Fe}$, we find

$$
\mu_{F e}=g \frac{e \hbar}{2 m_{p}} J=g \mu_{N} J
$$

where $\mu_{N}$ is the nuclear magneton. For the ${ }^{57} \mathrm{Fe}$ isotope ground state, the spin of the nucleus is $1 / 2$ [4].

$$
\mu_{F e}=g \mu_{N} J=5.75192609 \times 10^{-8} \mathrm{eV} \cdot \mathrm{T}^{-1}
$$

We can now find the magnetic potential energy of the magnetic moment. The energy is given by the dot product of the magnetic moment and the magnetic field:

$$
E=-\vec{\mu} \cdot \vec{B}
$$

where $E$ is the energy of the magnetic moment. Plugging in for $\mu$ gives us

$$
E=-\vec{\mu} \cdot \vec{B}=-\left(5.75192609 \times 10^{-8} \mathrm{eV} \cdot \mathrm{T}^{-1}\right) B
$$

The magnetic moment will have this magnetic potential energy when placed within the magnetic field. This value is also how much the energy of the atomic levels of the ${ }^{57} \mathrm{Fe}$ isotope will be shifted.

The directions of the magnetic moment and the magnetic field matter here; the orientation of the magnetic moment relative to the magnetic field will determine how the electron energy levels will split. The energy will be at its minimum when these are parallel and will be at its maximum when these point in opposite direction. The difference in energy between this minimum and maximum is

$$
\Delta E=2 \mu B \quad[6]
$$

\subsection{Dependence on T assuming magnetized iron foil}

For ${ }^{57} \mathrm{Fe}$ nuclei in an external magnetic field, there are two distinct states corresponding to alignment or anti-alignment of nuclear spin. As indicated earlier, these two states have different energies; thus, we can estimate the average nuclear polarization by computing the Boltzmann factors for the relative probabilities of the aligned state, $E_{1}$, and the anti-aligned state, $E_{2}$. 


$$
\frac{\operatorname{Pop}\left(E_{1}\right)}{\operatorname{Pop}\left(E_{2}\right)}=\exp \left(\frac{E_{2}-E_{1}}{k T}\right)=\exp \left(\frac{\Delta E}{k T}\right)
$$

Polarization, $P$, is defined as follows:

$$
P=\frac{\operatorname{Pop}\left(E_{1}\right)-\operatorname{Pop}\left(E_{2}\right)}{\operatorname{Pop}\left(E_{1}\right)+\operatorname{Pop}\left(E_{2}\right)}
$$

Thus $P=1$ implies all of the nuclei are aligned, while $P=-1$ implies they are all anti-aligned. A little algebra gives

$$
P=\frac{e^{\frac{\Delta E}{k T}}-1}{e^{\frac{\Delta E}{k T}}+1}
$$

\subsection{Emission \& angular momentum conservation}

The total angular momentum $\vec{J}$ is defined as the sum of the spin angular momentum, $\vec{s}$, and the orbital angular momentum, $\vec{l}$.

$$
\vec{J}=\vec{s}+\vec{l}
$$

The decay of a radioactive atom by gamma emission changes the atomic state from an excited state to a lower energy state with an emission of a photon. In the decay of the atomic state and the emission of the photon, both the total angular momentum and the total parity of the system must be conserved. These two conservation laws control the possible properties of the emitted photon.

\subsubsection{Conservation of angular momentum}

From conservation of total angular momentum,

$$
\vec{J}_{\text {initial }}=\vec{J}_{\text {final }}+\vec{J}_{\gamma}
$$

where $\vec{J}_{\text {initial }}$ is the angular momentum vector of the initial atomic state, $\vec{J}_{\text {final }}$ is the angular momentum vector of the decayed atomic state, and $\vec{J}_{\gamma}$ is the angular momentum of the emitted photon.

Quantum mechanics states that angular momentum is quantized. The magnitude of the angular momentum $\vec{J}$

is $\sqrt{j(j+1)} \hbar$, where $j=0,1 / 2,1,3 / 2, \ldots$ are the possible values for the total angular momentum quantum number. Using this along with the quantum laws of angular momentum addition, we find the possible values of the total angular momentum of the final state:

$$
j_{\text {decayed }}=\left|j_{\text {initial }}-j_{\gamma}\right|,\left|j_{\text {initial }}-j_{\gamma}\right|, \ldots, j_{\text {initial }}+j_{\gamma}-1, \quad j_{\text {initial }}+j_{\gamma}
$$

This shows that the possible values of $j_{\text {decayed }}$ increase from $\left|j_{\text {initial }}-j_{\gamma}\right|$ to $j_{\text {initial }}+j_{\gamma}$.

Only one component of the total angular momentum vector can possess a definite value in quantum mechanics. In our case we will choose the axis of quantization of the system to lie along the $z$-axis defined by the path of the photon between emitter and absorber. The possible values for the $z$ component of the total angular momentum are $m \hbar$, where $m$ is the magnetic quantum number with values ranging from $-j$ to $j$ by increments of 1 . So, conservation of angular momentum in the $z$-direction can be expressed as

$$
m_{\text {final }}=m_{\text {initial }}-m_{\gamma}
$$

\subsubsection{Conservation of parity}

A parity transformation consists in the inversion of all of the coordinate axes. The parity quantum number associated with this transformation on a quantum mechanical state, represented by $\pi$, can have only two values: either -1 or 1 . The electromagnetic interaction which generates and absorbs the photon conserves parity symmetry, and therefore the total parity of the system is a conserved quantum number. Parity is a multiplicative quantum number. Conservation of parity states therefore implies that the initial parity must be equal to the product of the parity of the final atomic state and the parity of the emitted photon

$$
\pi_{\text {initial }}=\pi_{\text {final }} \cdot \pi_{\gamma}
$$

The parity of a particle moving in an atomic orbital depends on the orbital angular momentum azimuthal quantum number $l$.

$$
\pi=(-1)^{l}
$$


Therefore conservation of parity in the case of an emitted photon may be expressed as

$$
(-1)^{l_{\text {initial }}}=(-1)^{l_{\text {final }}} \cdot \pi_{\gamma}
$$

We now have the selection rules which determine the possible spins and parities of the emitted photon in terms of the properties of the initial and final states of the nucleus.

\subsubsection{Application of conservation laws to the emission of a photon by polarized ${ }^{57} \mathrm{Fe}$ nuclei}

We now apply these general rules to the specific case of the particular transition of interest to this work. The emission of a photon by the decay of ${ }^{57} \mathrm{Fe}$ is a mixed electric quadrupole transition and magnetic dipole transition. The emitted photon has a net angular momentum, $j_{\gamma}$, of $l$ and an intrinsic parity, $\pi_{\gamma}$, of $(-1)^{l}$ for $E_{2}$ transition and $(-1)^{l}+1$ for $M_{1}$ transition.

So, substituting into Eqn.(46), conservation of parity gives

$$
(-1)^{l_{\text {initial }}}=(-1)^{l_{\text {final }}} \cdot(-1)^{l_{\gamma}+1}
$$

The decay of the excited state of ${ }^{57} \mathrm{Fe}$ involves the decay from an initial atomic state with an azimuthal quantum number of $\pm 3 / 2$ to a final state with an azimuthal quantum number of $\pm 1 / 2$. Through substitution, this gives

$$
(-1)^{ \pm \frac{3}{2}}=(-1)^{ \pm \frac{1}{2}} \cdot(-1)^{l_{\gamma}+1}
$$

The photon has an orbital angular momentum number $l_{\gamma}$ of 0 . The photon's parity is then calculated to be $(-1)^{1}$, or -1 , which agrees with the known intrinsic parity of the photon. The photon has total angular momentum, $\vec{J}_{\gamma}$, of $\mathrm{s}$, as $l_{\gamma}$ equals zero, where $s=1$.

This means that the spin and parity of the emitted photon agree with the conservation laws and produces a photon with a known angular momentum dependent upon the photon's spin.

The polarization of the emitted photon can then be either aligned or anti-aligned with the magnetic field based upon the alignment of the iron sample. The experimental question then becomes how to reverse this polarization. We describe the available techniques for doing this.

\subsection{Reversal of angular momentum using magnetic resonance}

Nuclear magnetic resonance (NMR) can be used to invert the spin of the excited iron, thereby changing the polarization of the emitted gamma ray. A powerful static magnetic field can be used to polarize the iron nuclei along the direction of the field. Since the decay product of ${ }^{57} \mathrm{Co}$ is ${ }^{57} \mathrm{Fe}^{*}$ which has a spin of $3 / 2$, the Zeeman effect occurs and the energies of the different states are split. A slight perturbation to the system in the form of a radio wave at the correct frequency can flip the spin from aligned to antialigned with the field.

The frequency of precession is the Larmor angular frequency described by the Larmor Equation:

$$
\omega=\gamma \mathbf{H} \quad \text { and } \quad \gamma=\frac{\boldsymbol{\mu}}{I}=\frac{g \beta}{\hbar}
$$

where $\gamma$ is the gyromagnetic ratio (1.382 MHz/T), $I$ is the nuclear angular momentum, $\mathbf{H}$ the magnetic field strength, $g$ the nuclear g-factor unique to the nuclei (3.276 for $\left.{ }^{57} \mathrm{Fe}^{*}\right)$, and $\beta=3.152451 \times 10^{-8} \mathrm{eV} / \mathrm{T}$ is the unit of magnetic moment called the nuclear magneton.

Nuclei at room temperature will be randomly oriented according to a Boltzmann distribution:

$$
\frac{N^{-}}{N^{+}}=e^{-\frac{\Delta E}{k T}}
$$

However, when a magnetic field $\left(B_{0}\right)$ is applied, alignment with the field becomes thermodynamically favored. Energy levels in the field are thus:

$$
\Delta E=-\mu B_{0}=-m \hbar \gamma B_{0}, m=-\frac{3}{2},-\frac{1}{2}, \frac{1}{2}, \text { or } \frac{3}{2}
$$

Substituting for $\gamma, \Delta E=g \beta B_{0}$ and using the energy of a photon $E=f c$, the required photon frequency is the following:

$$
f=\frac{g \beta B_{0}}{\hbar}
$$

One must therefore choose a temperature and a magnetic field environment which both leads to a large nuclear polarization and also a practical frequency for spin reversal. Natural iron in the ferromagnetic state has a very large internal magnetic field which makes the hyperfine levels visible at room temperature, and the hyperfine spectra have been measured for a very broad range of iron-containing materials. However, it is not practical to flip this internal magnetic field by external means. Therefore, in this material, one would need to induce spin flips by absorption of photons of the right frequency as shown above [5].

This method is the usual technique used to flip nuclear spins and is used in magnetic resonance imaging. However for our experiment it comes with a severe disadvantage. The problem is that the Mössbauer photon now has an extra contribution to its energy from the extra energy of the transition between the magnetic sublevels. Therefore, the photon is no longer in resonance with the absorber. This would not be a problem if the exact same energy level splitting of magnetic sublevels was present in the absorber. However, this equality might demand a level of accuracy of the magnetic fields in the two separate samples, which is difficult to achieve. We are then led to consider an alternative method for sensing the angular momentum dependence of the gravitational redshift of the photon.

\subsection{Use of the Faraday effect}

Consider producing a photon which is not in a definite state of spin angular momentum about the $z$-axis, but is rather in an 
equal quantum superposition of the two angular momentum projections along $z$. Such a photon state has a name: it is called linear polarization. We could therefore conduct the spin-dependent experiment in a slightly different way. Assume we can arrange the Mössbauer transition to generate such a linearly polarized photon state. If there is a spin-dependence to the gravitational redshift, the plane of polarization of this radiation will rotate from its original orientation as the energy, and therefore the quantum mechanical phase of the two different components of the photon state become slightly different. The sign of the effect flips when the photons travel in the opposite direction of the gravitational field. Therefore, we can alternatively search for a rotation of the plane of polarization of the photons instead.

\subsection{Other possible nuclei}

For the original Pound-Rebka experiment, they used ${ }_{26}^{57} \mathrm{Fe}$, but very little is stated in the Pound-Rebka experiment as to why specifically this isotope of iron was chosen, and what led to that decision. In this section, we explore the possibility of using a different nucleus to conduct our experiment with higher accuracy. To consider this, we must take every nucleus that possesses a Mossbauer transition and take a survey of their properties to find the best viable option while also considering practical availability. To do this we construct a spreadsheet detailing each isotope with its abundance (\%), excited state energy (keV), and excited half-life (ns). We also make note of any peculiarities such as radioactivity or unknown values, as these unknowns would cause unpredictable results in the experimental process. Using this spreadsheet one can filter the results using a figure of merit which applies values to each transition, isotope, and nucleus in order to determine its comparative usefulness for our purpose. In this case we take the product of the excited state energy times the excited half-life times the square root of the isotopic abundance,

$$
E_{\text {excited }} \cdot t_{1 / 2} \cdot \sqrt{A}
$$

where $E$ is the excited state, $t$ is the half-life of the excited state, and $A$ is the isotopic abundance. This value is proportional to the ratio of the energy width of the resonance, $\delta E$, and the gamma energy, $E$. Assuming we need a minimum ratio, first we set the minimum value for the figure of merit to be the minimum value which was calculated from the ${ }^{57} \mathrm{Fe}$ (approximately 200). Then, we sort through the results from other nuclei, and disregard any that either has no second transition, or for which the second transition does not meet the results set by the figure of merit. This leaves only ${ }_{26}^{57} \mathrm{Fe}$ and ${ }_{32}^{73} \mathrm{Ge}$ as viable options, and Ge gives a figure of merit upwards of 10,000 , nearly 100 times that found for Fe. This is mainly due to its very long half-life.

\section{CONCLUSION}

In this collective research project, we have considered some of the important issues of principle and some of the relevant practical details for a future experiment to search for the possible spin dependence of the gravitational redshift using the Mössbauer effect with polarized photons. We have described the important physical effects involved in the phenomena themselves and outlined some of the important issues that must be considered for a real experiment. We conclude that there is no issue of principle or safety issue which forbids such an experiment from being realized. We have identified some of the important problems that must be addressed to realize the new feature of the proposed experiment, namely the measurement of the polarization dependence. We have identified a possible new approach based on the use of transversely polarized light rotation which could be a good choice for this experiment, as it is possible to measure this rotation of the plane of polarization without changing the energy of the source of the absorber (as required in methods which employ a spin flip of some type).

Two main steps need to be taken to realize this experiment. First, one must analyze the various sources of systematic errors with one of the methods in mind and convince oneself that there is no experimental control parameter that is impossible to achieve. In addition, there must be enough progress on the theory of exotic spin-dependent effects in gravity to have some idea of the expected size of a possible effect as this can strongly influence the choice of a specific nucleus, although in our case we used data from the very well-understood transition in iron that has been so heavily used in Mössbauer studies.

\section{AUTHOR INFORMATION}

All correspondence should be sent to the first authors: taspayne@indiana.edu and nkschlos@indiana.edu.

\section{REFERENCES}

[1] Davis J.C., Gullikson E.M., Henke B.L. (1993). $X$-ray Interactions: Photoabsorption, Scattering, Transmission, and Reflection at $E=50-30,000 \mathrm{eV}$, $Z=1-92$. Atomic Data and Nuclear Data Tables 54 . Academic Press, p. 181-342.

[2] Dunford B.H., Dolphin D., Raymond K.N., Sieker L. (1981). The Biological Chemistry of Iron: A Look at the Metabolism of Iron and Its Subsequent Uses in Living Organisms. Proceedings of the NATO Advanced Study Institute held at Edmonton, Alberta, Canada, Aug. 13.

[3] Dunham, W.R. et al. (1977). Added Precision in ${ }^{57} \mathrm{Fe}$ Mössbauer Spectroscopy. University of Michigan.

[4] Freude, D. (2006). Nuclear Magnetic Resonance. In Spectroscopy for Physicists.

[5] Fultz B. (2011). "Mössbauer Spectrometry," in Characterization of Materials. Kaufmann E., editor. New York (NY): John Wiley \& Sons.

[6] Gibb, T.C. (1976). Principles of Mössbauer Spectroscopy. London: Chapman \& Hall.

[7] Gullikson E.M. (2001). Section 1.7 "Atomic Scattering Factors”. In X-Ray Data Booklet, edited by Attwood 
D.T., Vaughan D. Berkeley: Lawrence Berkeley National Laboratory.

[8] Kittel C. (2004). Introduction to Solid State Physics ( 8 ed.). New York (NY): John Wiley \& Sons.

[9] Lawrence Berkeley Lab Center for X-Ray Optics. $X$-Ray Interactions With Matter: Introduction, atomic scattering files, and $x$-ray properties of the elements.

[10] Nalwa, H. S. (2001). Handbook of Thin Films, FiveVolume Set (Vol. 5). Burlington: Elsevier Science \& Technology.

[11] National Institute of Standards and Technology. Fundamental Physical Constants: Nuclear Magneton in $\mathrm{eV} / \mathrm{T}$.

[12] National Institute of Standards and Technology. XCOM: Photon Cross Sections Database.

[13] National Institute of Standards and Technology. Tables of X-Ray Mass Attenuation Coefficients and Mass Energy-Absorption Coefficients from $1 \mathrm{keV}$ to $20 \mathrm{MeV}$ for Elements $\mathrm{Z}=1$ to 92 and 48 Additional Substances of Dosimetric Interest.

[14] National Institute of Standards and Technology. X-Ray Form Factor, Attenuation, and Scattering Tables.

[15] Pound R.V., Rebka G.A. (1960). Apparent Weight of Photons. Phys. Rev. Lett. 4. American Physical Society, p. 337-341.

[16] Shultis, J., Faw, R. (2007). Fundamentals of nuclear science and engineering. Boca Raton, FL: CRC Press.

[17] Wolter, H. (1952). Spiegelsysteme streifenden Einfalls als abbildende Optiken für Röntgenstrahlen. Annalen der Physik, vol. 10, p. 94-114. 